\title{
Identification of Long Noncoding RNA APOC1P1 as an Oncogene in Clear Cell Renal Cell Carcinoma
}

\author{
Chong Sun $\mathbb{D}^{1}$, Zhonghan Zhou $\mathbb{D}^{2}$, Haoqing Shi $\mathbb{D}^{2},{ }^{2}$ Fangzhou Li $\mathbb{D}^{2},{ }^{2}$ and Guiming Zhang $\mathbb{D}^{2}$ \\ ${ }^{1}$ Department of Spinal Surgery, The Affiliated Hospital of Qingdao University, Qingdao, China \\ ${ }^{2}$ Department of Urology, The Affiliated Hospital of Qingdao University, Qingdao, China \\ Correspondence should be addressed to Guiming Zhang; zhangguiming9@126.com
}

Received 16 July 2019; Revised 7 October 2019; Accepted 24 October 2019; Published 1 December 2019

Academic Editor: Giuseppe Biondi-Zoccai

Copyright (C) 2019 Chong Sun et al. This is an open access article distributed under the Creative Commons Attribution License, which permits unrestricted use, distribution, and reproduction in any medium, provided the original work is properly cited.

\begin{abstract}
Renal cell carcinoma (RCC) is one of the most common genitourinary cancers worldwide. Previous evidence shows that long noncoding RNA (LncRNA) APOC1P1 plays an important role in cancer development. However, the role of LncRNA APOC1P1 in ccRCC remains to be explored. LncRNA APOC1P1 expression in 283 ccRCC tissues and 30 normal kidney tissues was detected by quantitative real-time PCR, and its prognostic association with ccRCC was assessed by the Kaplan-Meier method and Cox proportional hazard model. Cell proliferation, apoptosis, migration, and invasion were determined in RCC cells with downregulation of LncRNA APOC1P1 expression. LncRNA APOC1P1 expression was increased in ccRCC tissues compared with normal kidney tissues $(P<0.001)$. Its expression was higher in the Fuhrman grade III and IV group than in the Fuhrman grade I and II group $(P<0.05)$ and significantly upregulated in the advanced stage group $(P<0.05)$. Kaplan-Meier analyses revealed that elevated LncRNA APOC1P1 expression was significantly associated with poor overall survival $(P<0.05)$ but may not be an independent prognostic factor. Knockdown of LncRNA APOC1P1 inhibited cell proliferation, induced apoptosis, and arrested cells at the G1/S phase $(P<0.05)$. Silencing of LncRNA APOC1P1 also led to decreased cell migration and invasion $(P<0.05)$. LncRNA APOC1P1 acts as an oncogene, plays an important role in ccRCC development, and can be considered a prognostic biomarker and therapeutic target in ccRCC patients.
\end{abstract}

\section{Introduction}

Renal cell carcinoma (RCC) is the most lethal of the common urological cancers and was responsible for $2.2 \%$ of newly diagnosed cancers and $1.8 \%$ of deaths in $2018[1,2]$. Historically, clear cell RCC (ccRCC) is the most common subtype and accounts for $80-90 \%$ of all RCC cases [3,4]. Despite the striking progress in cancer detection and treatment, the overall survival (OS) rate in RCC still remains poor $[5,6]$. Approximately $1 / 3$ of RCC patients have evidence of metastases at presentation, and the 5-year age-standardized relative survival rate for stage IV disease is $6 \%$ compared with $84 \%$ for stage I disease [7]. Thus, it is essential to clarify the mechanisms for RCC carcinogenesis and to identify novel biomarkers that can predict its prognosis.

Long noncoding RNAs (LncRNAs) comprise a class of noncoding RNAs that are 200 to 100,000 nucleotides in length without protein-coding capacity $[8,9]$. Studies have revealed that LncRNAs play an important role in tumorigenesis and metastasis [10-12]. LncRNA apolipoprotein C-1 pseudogene 1 (LncRNA APOC1P1), which is located at $19 \mathrm{q} 13.2$ between APOC-I and APOC-IV, was shown to inhibit apoptosis by decreasing $\alpha$-tubulin acetylation in breast cancer [13]. However, the role of LncRNA APOC1P1 in ccRCC is yet to be explored. In this study, we aimed to determine the expression of LncRNA APOC1P1 in ccRCC and its impact on patients' survival. As well, the biological functions of LncRNA APOC1P1 in RCC cells were also investigated.

\section{Materials and Methods}

2.1. Patients and Specimens. A total of 283 ccRCC specimens and 30 adjacent normal renal tissues were collected from 
patients who underwent radical or partial nephrectomy between January 2007 and December 2010 at the Department of Urology, The Affiliated Hospital of Qingdao University. All specimens were confirmed by postoperative pathological analysis. Tumor staging and grading were confirmed according to the AJCC TNM 2010 classification system and WHO/ISUP 2004 system, respectively. None of the patients received chemotherapy, radiotherapy, or targeted therapy before surgery. Tissues were stored at $-80^{\circ} \mathrm{C}$ immediately after surgical removal for later analysis. Patients were regularly followed up in the clinic or by telephone until June 2018 or date of death. The study protocol was approved by the Ethics Committee of The Affiliated Hospital of Qingdao University, and written informed consent was obtained from all patients before surgery.

2.2. RNA Extraction and Quantitative Real-Time PCR ( $q R T$ $P C R)$. The RNA extraction was performed right before the analyses, and RNA quality control was performed by the Thermo Scientific NanoDrop ${ }^{\mathrm{TM}} 1000$ Spectrophotometer. TRIzol reagent (Thermo Fisher Scientific Inc., Waltham, MA, USA) was used to extract total RNA from tissues or cells according to the manufacturer's protocols and subjected to reverse transcription using a RevertAid First Strand cDNA Synthesis Kit (Thermo Fisher Scientific Inc.). Then, qRTPCR was performed using a Power SYBR Green PCR Master Mix (Thermo Fisher Scientific Inc.) and an ABI 7900HT Fast Real-Time PCR system (Applied Biosystems, Foster City, CA, USA). Relative expression values of LncRNA APOC1P1 were normalized to $\beta$-actin. The gene-specific primers sequences were as follows: LncRNA APOC1P1, forward, $5^{\prime}$ GGTCCTGGTGGTGGTTCTGTC- ${ }^{\prime}$, reverse, $5^{\prime}$-CTCCTT CACTTTCCGAAATGTCTC- $3^{\prime} ; \beta$-actin, forward, $5^{\prime}$-AC CGAGCGCGGCTACAG- $3^{\prime}$, reverse, $5^{\prime}$-CTTAATGTCAC GCACGATTTCC- $3^{\prime}$. All experiments were carried out in triplicate.

2.3. Cell Culture and siRNA Transfection. Human RCC cell lines (A498, 786-O, ACHN, and Caki-1) and an immortalized normal human renal tubular epithelial cell line (HK-2) were purchased from The Institute of Cell Research of the Chinese Academy of Sciences (Shanghai, China). A498, 786-O, and HK-2 cells were cultured in RPMI-460 medium (Hyclone; GE Healthcare Life Science, Logan, UT, USA). ACHN and Caki-1 cells were grown in MEM medium and McCoy's 5A medium (Gibco; Thermo Fisher Scientific Inc.), respectively. All media were supplemented with $10 \%$ fetal bovine serum (FBS; Hyclone; GE Healthcare Life Science). Cells were cultured at $37^{\circ} \mathrm{C}$ with $5 \% \mathrm{CO}_{2}$. Three small interfering RNAs (siRNAs) targeting LncRNA APOC1P1 (siRNA1, siRNA2, and siRNA3) were designed by Guangzhou RiboBio Co. Ltd. (Guangzhou, China). siRNA transfection was conducted with a Lipofectamine 2000 Reagent (Invitrogen, Carlsbad, CA, USA) in accordance with the manufacturer's protocols. The efficiency of knockdown was tested $72 \mathrm{~h}$ after transfection.

2.4. Cell Proliferation, Cell Cycle, and Apoptosis Assays. Cell proliferation capacity was determined using a Cell Count
Kit-8 (CCK-8 assay; Dojindo, Shanghai, China). Briefly, cells were plated in 96-well culture plates at a density of $1 \times 10^{4}$ cells/well and cultured in fresh medium mixed with CCK-8 at a ratio of 10:1 for $2 \mathrm{~h}$. The cell density was detected with an enzyme-linked immunosorbent assay microplate reader at $490 \mathrm{~nm}$.

Cell cycle assays were performed using flow cytometry. Briefly, cells were fixed with $75 \%$ cold ethanol overnight and then washed with phosphate-buffered saline. Propidium iodide $(50 \mu \mathrm{g} / \mathrm{ml})$ mixed with RNase was added for DNA staining. Then, cells were analyzed by flow cytometry (Beckman Coulter, Brea, CA, USA).

Cell apoptosis was determined by flow cytometry, too. Briefly, after DNA staining with $5 \mu \mathrm{l}$ of FITC-Annexin V and $5 \mu \mathrm{l}$ of propidium iodide (BD Biosciences, Franklin Lakes, NJ, USA), cells were incubated at room temperature in the dark for $15 \mathrm{~min}$. An additional $400 \mu \mathrm{l}$ of binding buffer was then added before analysis by flow cytometry.

2.5. Wound Healing and Transwell Cell Invasion Assays. The in vitro wound healing assays were conducted to detect the cell migration capacity. Briefly, cells were seeded in 6-well plates to form a confluent monolayer. An artificial homogeneous scratch wound was made using a sterile $200 \mu \mathrm{l}$ pipette tip. After incubation for 0,24 , and $72 \mathrm{~h}$, images of the cells were randomly captured under an inverted microscope (IX51; Olympus Corporation Co. Ltd., Tokyo, Japan) at $\times 100$ magnification. Migratory capacity was measured as relative migratory distance. All experiments were carried out in triplicate.

Transwell assays were performed to determine the cell invasion capacity using 24-well Transwell chambers (Corning, NY, USA, No.353097, $8 \mu \mathrm{m}$ ). First, the upper chambers were precoated with $60 \mu \mathrm{l}$ of Matrigel (BD Biosciences, Franklin Lakes, NJ, USA). Cells were then seeded in the upper chambers in serum-free medium at a density of $4 \times$ $10^{4}$ cells/chamber, while medium supplemented with $10 \%$ FBS was added to the lower chambers. After $48 \mathrm{~h}$ of incubation at $37^{\circ} \mathrm{C}$, inserts were stained with $0.5 \%$ crystal violet (Shanghai Macklin Reagent Co. Ltd., Shanghai, China) for $30 \mathrm{~min}$. The numbers of invaded cells were counted in randomly selected fields under a microscope. Invasion capacity was measured as relative cell counts. All experiments were carried out in triplicate.

2.6. Statistical Analysis. SPSS 19.0 software (IBM Corporation, Armonk, NY, USA) was used for statistical analyses. Continuous variables were expressed as mean \pm standard deviation (SD). Student's $t$-test and the chi-square test were performed for continuous and categorical variables, respectively. The receiver operating characteristic (ROC) curve was applied to calculate the area under the ROC curve (AUC) for evaluating the diagnostic efficacy of LncRNA APOC1P1. The Kaplan-Meier method was used to detect the influence of LncRNA expression on survival. Univariate and multivariate analyses were carried out by the Cox proportional hazard model. Values of $P<0.05$ were considered statistically significant. 


\section{Results}

3.1. LncRNA APOC1P1 Expression in ccRCC Tissues and Normal Renal Tissues. The clinicopathological features of the patients are presented in Table 1 . Patients with ccRCC were divided into low $(n=142)$ and high $(n=141)$ expression groups based on the median value $(0.40)$ of relative LncRNA APOC1P1 expression, and clinicopathologic features were compared between the two groups. As summarized in Table 1, increased LncRNA APOC1P1 expression significantly correlated with the Fuhrman grade, T-stage, and Eastern Cooperative Oncology Group (ECOG) grade $(P<0.05)$. However, there was no significant correlation between LncRNA APOC1P1 expression and other clinicopathologic features such as age, gender, BMI, smoking and drinking status, history of hypertension, and diabetes $(P>0.05)$. LncRNA APOC1P1 expression was increased in ccRCC tissues compared with adjacent normal renal tissues $(P<0.001$, Figure 1(a)). Furthermore, its expression was higher in the Fuhrman grade III-IV group than in the Fuhrman grade I-II group $(P<0.05$, Figure $1(\mathrm{~b}))$ and significantly upregulated in the advanced ccRCC group compared with the localized ccRCC group $(P<0.05$, Figure $1(\mathrm{c}))$. These findings suggest that LncRNA APOC1P1 may play a tumorpromoting role in ccRCC.

The diagnostic efficacy of LncRNA APOC1P1 was evaluated. The ROC curve analysis revealed that AUC was 0.853 (95\%CI $=0.738$ to $0.931, P<0.001$, Figure $1(d)$ ). When the cutoff value $=-0.442$, the diagnostic sensitivity $(73.3 \%)$ and specificity $(93.3 \%)$ reached their peak value, indicating that LncRNA APOC1P1 has clinical significance for the diagnosis of ccRCC.

3.2. Influence of LncRNA APOC1P1 Expression on Survival. The follow-up period for the ccRCC patients ranged from 10 to 127 months (mean: 82.2 months). Kaplan-Meier analysis revealed that elevated LncRNA APOC1P1 expression was significantly associated with poor OS $(P<0.05$, Figure 1(e)). In the univariate Cox regression, Fuhrman stage, TNM stage, Eastern Cooperative Oncology Group (ECOG) grade, and LncRNA APOC1P1 expression were prognostic factors in ccRCC patients (Figure 2(a)). However, the multivariate Cox regression indicated that LncRNA APOC1P1 expression may not be an independent prognostic factor (Figure 2(b)).

3.3. Effect of LncRNA APOC1P1 Knockdown on Proliferation, Apoptosis, and Cell Cycle. We measured the expression of LncRNA APOC1P1 in four human ccRCC cell lines (A498, 786-O, ACHN, and Caki-1) and an immortalized normal human renal tubular epithelial cell line (HK-2) by qRTPCR. As shown in Figure 3(a), there was higher expression of LncRNA APOC1P1 in all four ccRCC cell lines compared with HK-2 cells $(P<0.05)$, consistent with the expression pattern of LncRNA APOC1P1 in the tissue specimens. A498 cells exhibited the highest LncRNA APOC1P1 expression $(P<0.05)$, and thus, the A498 cell line was selected for further analyses.
TABLE 1: Clinicopathological features of patients with ccRCC.

\begin{tabular}{|c|c|c|c|c|c|}
\hline \multirow[t]{2}{*}{ Parameters } & \multirow[t]{2}{*}{ Group } & \multirow[t]{2}{*}{ Total } & \multicolumn{2}{|c|}{$\begin{array}{l}\text { LncRNA } \\
\text { APOC1P1 } \\
\text { expression }\end{array}$} & \multirow[t]{2}{*}{$P$ value } \\
\hline & & & Low & High & \\
\hline \multirow{2}{*}{ Age } & $<60$ & 180 & 85 & 95 & \multirow[t]{2}{*}{0.217} \\
\hline & $\geq 60$ & 103 & 57 & 46 & \\
\hline \multirow{2}{*}{ Gender } & Male & 194 & 96 & 98 & \multirow[t]{2}{*}{0.798} \\
\hline & Female & 89 & 46 & 43 & \\
\hline \multirow{2}{*}{ BMI } & $<24$ & 128 & 68 & 60 & \multirow[t]{2}{*}{0.404} \\
\hline & $\geq 24$ & 155 & 74 & 81 & \\
\hline \multirow{2}{*}{ Smoking } & No & 223 & 111 & 112 & \multirow[t]{2}{*}{0.885} \\
\hline & Yes & 60 & 31 & 29 & \\
\hline \multirow{2}{*}{ Alcohol } & No & 259 & 131 & 128 & \multirow[t]{2}{*}{0.676} \\
\hline & Yes & 24 & 11 & 13 & \\
\hline \multirow{2}{*}{ Hypertension } & No & 184 & 88 & 96 & \multirow[t]{2}{*}{0.319} \\
\hline & Yes & 99 & 54 & 45 & \\
\hline \multirow{2}{*}{ Diabetes } & No & 211 & 111 & 100 & \multirow[t]{2}{*}{0.174} \\
\hline & Yes & 72 & 31 & 41 & \\
\hline \multirow{2}{*}{ Fuhrman grade } & $\mathrm{I} / \mathrm{II}$ & 133 & 50 & 83 & \multirow[t]{2}{*}{$<0.001$} \\
\hline & III/IV & 150 & 92 & 58 & \\
\hline \multirow{2}{*}{ T-stage } & $\mathrm{T} 1 / \mathrm{T} 2$ & 255 & 122 & 133 & \multirow[t]{2}{*}{0.027} \\
\hline & $\mathrm{T} 3 / \mathrm{T} 4$ & 28 & 20 & 8 & \\
\hline \multirow{2}{*}{ ECOG } & No & 266 & 127 & 139 & \multirow[t]{2}{*}{0.002} \\
\hline & Yes & 17 & 15 & 2 & \\
\hline
\end{tabular}

Transfection with LncRNA APOC1P1 siRNAs was performed to downregulate the expression of LncRNA APOC1P1, and transfection with siRNA2 and siRNA3 successfully decreased LncRNA APOC1P1 levels in A498 cells compared with the positive control group $(P<0.05$, Figure 3(b)). Moreover, siRNA2 exhibited the best transfection efficiency. Therefore, knockdown of LncRNA APOC1P1 was performed with siRNA2 to investigate its biological function.

Cell proliferation was evaluated by CCK- 8 assays after transfection with si-LncRNA APOC1P1 (in both the siRNA2 and siRNA3 groups). The results indicated that si-LncRNA transfection led to cell growth arrest in A498 cells compared with the si-NC group $(P<0.05$, Figure $3(\mathrm{c}))$.

Apoptosis and cell cycle analyses were performed at $48 \mathrm{~h}$ after siRNA transfection into A498 cells. As shown in Figure 3(d), the apoptosis rate was clearly increased after LncRNA APOC1P1 downregulation compared with the siNC transfection group $(P<0.05)$. In the cell cycle analyses, the proportion of cells in the G1-phase was significantly increased after transfection, while the proportion of cells in the S-phase was notably lower than that in the control group, suggesting that LncRNA APOC1P1 knockdown induced G1/S arrest $(P<0.05$; Figure 3(e)).

3.4. Effect of LncRNA APOC1P1 Knockdown on Cell Invasion and Migration. Wound healing and Transwell chamber assays were conducted to clarify the effect of LncRNA 


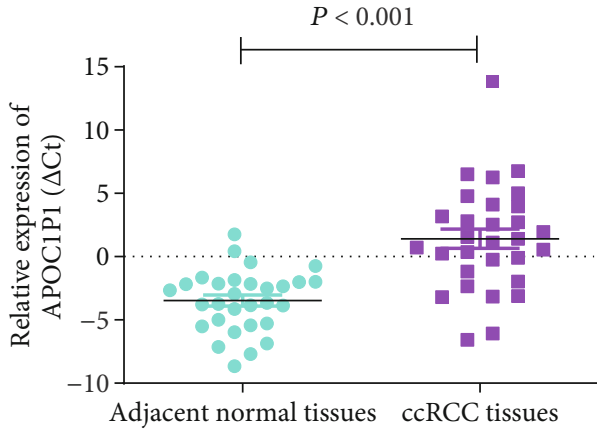

(a)

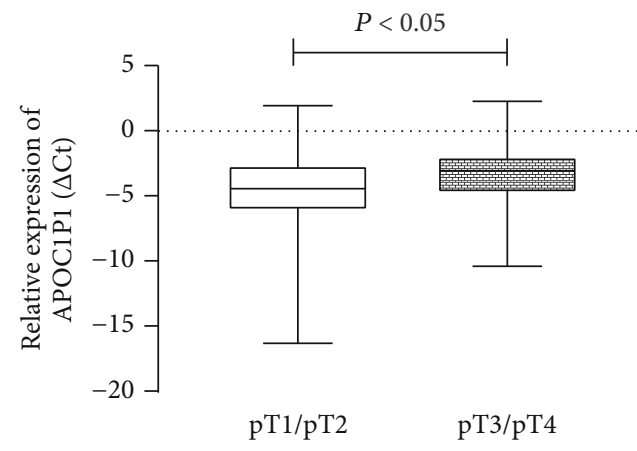

(c)

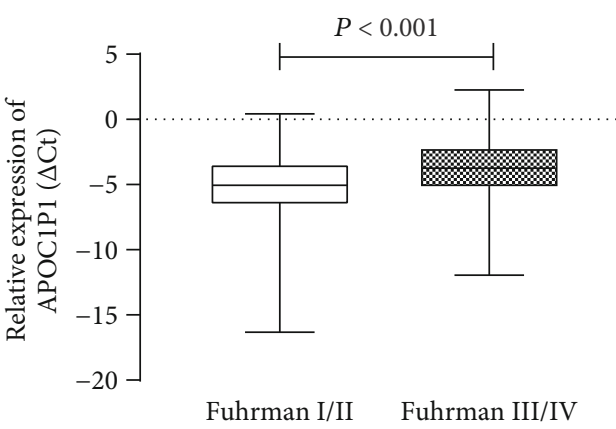

(b)

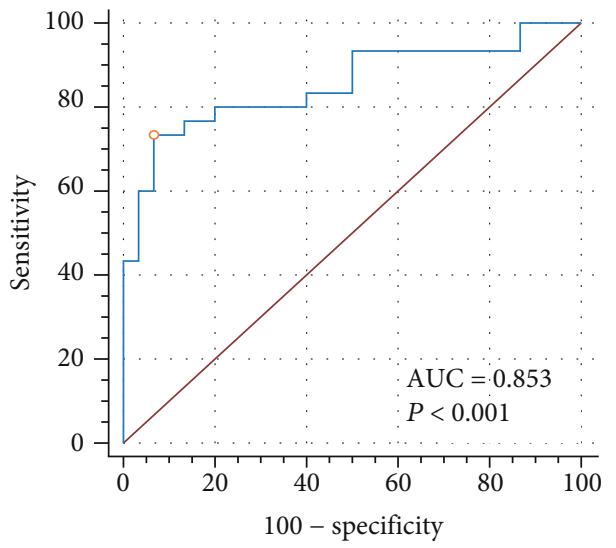

(d)

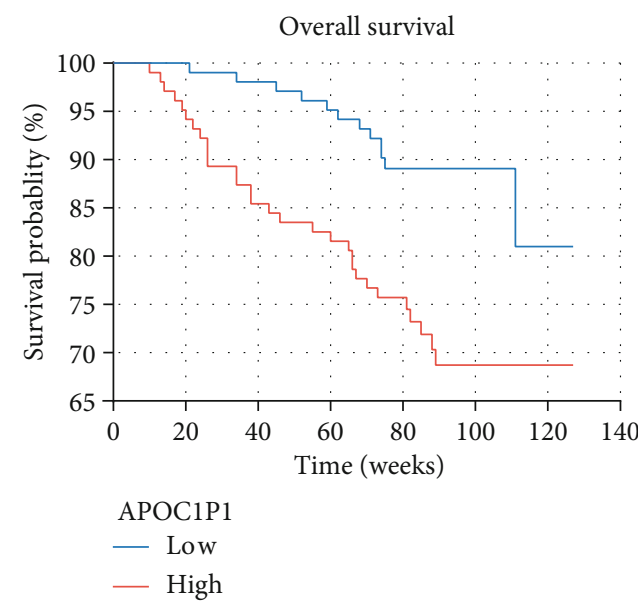

(e)

FIgURE 1: LncRNA APOC1P1 expression in ccRCC tissues and normal renal tissues and influence on overall survival. (a) LncRNA APOC1P1 expression in ccRCC tissues and adjacent normal renal tissues. (b) Comparison of LncRNA APOC1P1 expression with Fuhrman grade patterns. (c) Comparison of LncRNA APOC1P1 expression with stage patterns. (d) Assessment of the diagnostic efficacy of LncRNA APOC1P1 in ccRCC patients. (e) Influence of LncRNA APOC1P1 expression on overall survival. LncRNA APOC1P1 levels were quantified and normalized to $\beta$-actin. All experiments were carried out in triplicate. The data were presented by $x \pm s$.

APOC1P1 on cell migration and invasion. Compared with the si-NC group, cell migration was significantly inhibited after si-LncRNA APOC1P1 transfection, and cell invasive capacity was also markedly reduced $(P<0.05$, Figures 3(f) and $3(\mathrm{~g}))$. Compared with the si-NC group, cell invasive capacity was reduced after both siRNA2 and siRNA3 transfection, indicating that the off-target effect can be excluded $(P<0.05$, Figure 3(f)). Cell migration was also significantly inhibited after si-LncRNA APOC1P1 transfection $(P<0.05$, Figure 3(g)).

\section{Discussion}

Over 200,000 new RCC cases are diagnosed every year, and more than 1000,000 related deaths occur worldwide. In 2015, the International Agency for Research on Cancer 


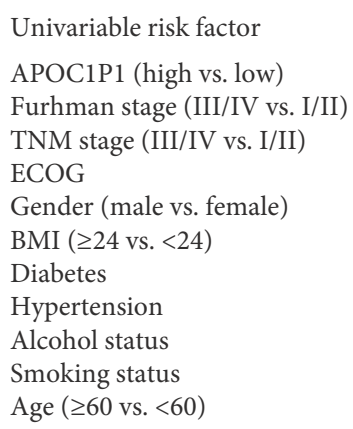

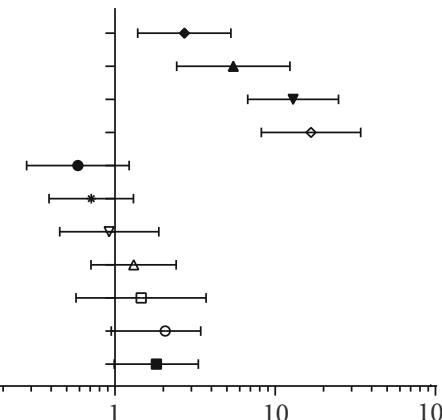

(a)

Multi variable risk factor
APOC1P1 (high vs. low)
Furhman stage (III/IV vs. 1/11)
TNM stage (III/IV vs. I/II)
ECOG
Gender (male vs. female)
BMI ( $\geq 24$ vs. $<24)$
Diabetes
Hypertension
Alcohol status
Smoking status
Age $(\geq 60$ vs. $<60)$

Multi variable risk factor APOC1P1 (high vs. low) Furhman stage (III/IV vs. 1/11) TNM stage (III/IV vs. I/II) ECOG

Gender (male vs. female) BMI ( $\geq 24$ vs. $<24)$ Diabetes Hypertension Age ( $\geq 60$ vs. $<60)$

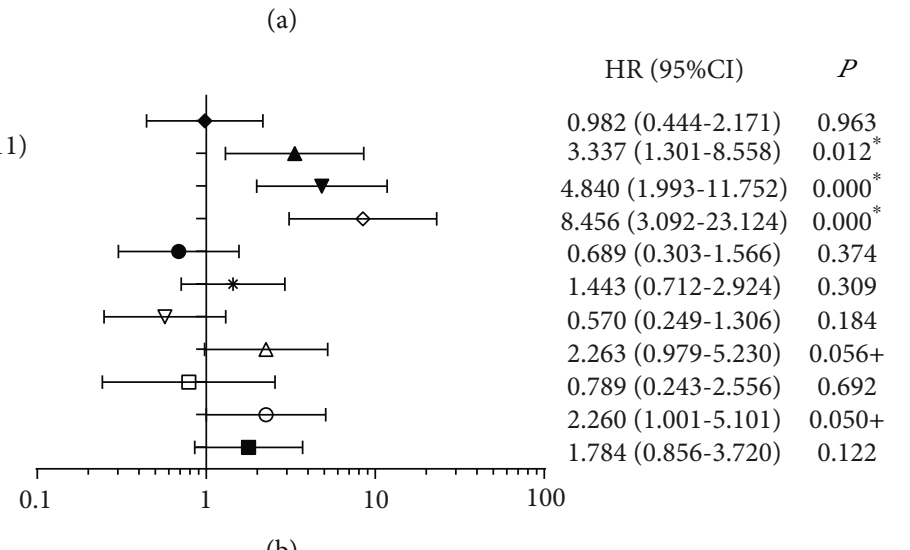

(b)

$\begin{array}{cc}\text { HR }(95 \% \mathrm{CI}) & P \\ 2.715(1.389-5.305) & 0.003^{*} \\ 5.486(2.434-12.362) & 0.000^{*} \\ 12.963(6.753-24.885) & 0.000^{*} \\ 16.754(8.206-34.209) & 0.000^{*} \\ 0.588(0.281-1.229) & 0.158 \\ 0.712(0.389-1.306) & 0.273 \\ 0.922(0.453-1.879) & 0.824 \\ 1.309(0.710-2.413) & 0.388 \\ 1.458(0.572-3.711) & 0.429 \\ 1.806(0.951-3.431) & 0.071+ \\ 1.813(0.990-3.321) & 0.054+ \\ & \end{array}$

$\begin{array}{cc}\text { HR }(95 \% \mathrm{CI}) & P \\ 0.982(0.444-2.171) & 0.963 \\ 3.337(1.301-8.558) & 0.012^{*} \\ 4.840(1.993-11.752) & 0.000^{*} \\ 8.456(3.092-23.124) & 0.000^{*} \\ 0.689(0.303-1.566) & 0.374 \\ 1.443(0.712-2.924) & 0.309 \\ 0.570(0.249-1.306) & 0.184 \\ 2.263(0.979-5.230) & 0.056+ \\ 0.789(0.243-2.556) & 0.692 \\ 2.260(1.001-5.101) & 0.050+ \\ 1.784(0.856-3.720) & 0.122 \\ & \end{array}$

FIgURe 2: Univariate (a) and multivariate (b) analyses of LncRNA APOC1P1 in ccRCC patients $\left({ }^{*} P<0.05 ;{ }^{+} P<0.10\right)$.

predicted a $22 \%$ increase in the number of people developing RCC by $2020[14,15]$. Thus, establishment of a sensitive and reliable molecular marker to aid in its diagnosis and treatment is in urgent need. In the last decades, many studies have shown that LncRNAs have multiple functions in a wide range of biological processes, such as the cell proliferation, cell apoptosis, cell cycle arrest, and cell migration and invasion [16]. Plenty of reports have investigated the diagnostic and prognostic value of LncRNAs in various cancers $[17,18]$.

Apolipoprotein C1 (APOC1), expressed primarily in the liver, encodes a member of the apolipoprotein $\mathrm{C} 1$ family which plays a crucial role in lipid metabolism [19-21]. It stimulates cell proliferation and prevents cell apoptosis [22]. APOC1P1, located at $19 \mathrm{q} 13.2$, is the pseudogene of APOC1. Generally, the antisense transcripts produced from pseudogenes can hybridize to corresponding mRNAs, forming dsRNAs cleaved by Dicer to endogenous siRNAs [23]. LncRNA APOC1P1 was found overexpressed in breast cancer, and its upregulation promotes cell proliferation [13]. However, the role of LncRNA APOC1P1 in ccRCC has not been reported. Therefore, the present study focused on the effects of LncRNA APOC1P1 during onset and progression of ccRCC.

It is reported that LncRNA APOC1P1 is overexpressed in breast cancer [13]. Similar results were observed in our study. The expression of LncRNA APOC1P1 was upregulated in ccRCC tissues compared with normal renal tissues. In addition, elevated expression was associated with higher Fuhrman grade and clinical stage. These results suggest that LncRNA APOC1P1 plays a role in tumor occurrence and development, which is inconsistent with previous study [13]. To assess its prognostic value in ccRCC patients, survival analysis was performed, revealing that LncRNA APOC1P1 expression was associated with worse OS. While the univariate Cox regression reached statistical significance, the multivariate Cox regression revealed that its expression may not be an independent prognostic factor. We attribute these findings to the close correlations between LncRNA APOC1P1 expression and clinicopathological factors, such as the Fuhrman stage, TNM stage, and ECOG grade. These conclusions require validation using large-sample, multicenter datasets. Furthermore, functional experiments in vitro were conducted to evaluate the biological roles of LncRNA APOC1P1 in ccRCC. Knockdown of LncRNA APOC1P1 expression inhibited cell proliferation, induced apoptosis, and arrested cells at G1/S phase. Silencing of LncRNA APOC1P1 also led to decreased cell migration and invasion. These findings suggest that LncRNA APOC1P1 may impact the clinicopathological features of ccRCC by affecting cell proliferation and invasiveness. A previous study revealed that LncRNA APOC1P1 can bind to tubulin and then increase $\alpha$-tubulin acetylation and inhibit apoptosis [13]. We assume that in ccRCC, LncRNA APOC1P1 might function in the same process. Clearly, further researches about the expression and function of LncRNA APOC1P1 in cancer initiation and progression are warranted.

\section{Conclusions}

In summary, we have identified elevated LncRNA APOC1P1 expression in ccRCC. Our findings indicated that LncRNA 


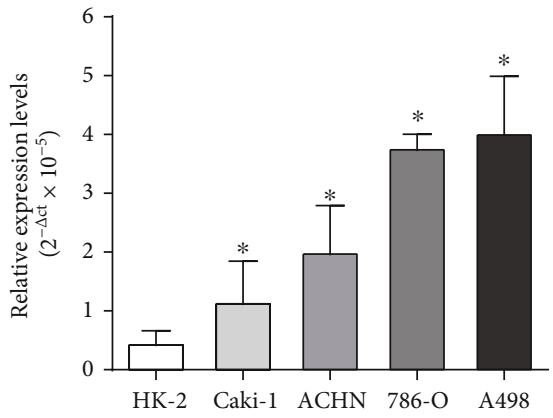

(a)

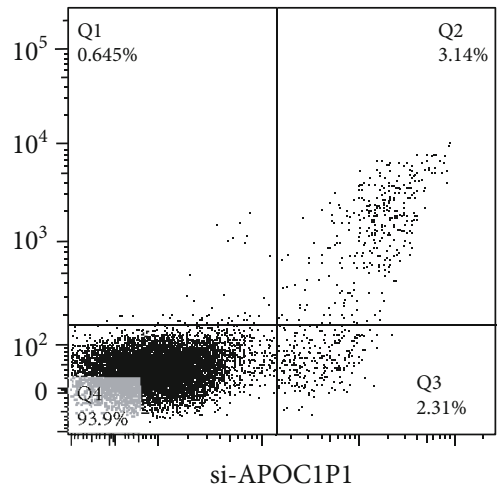

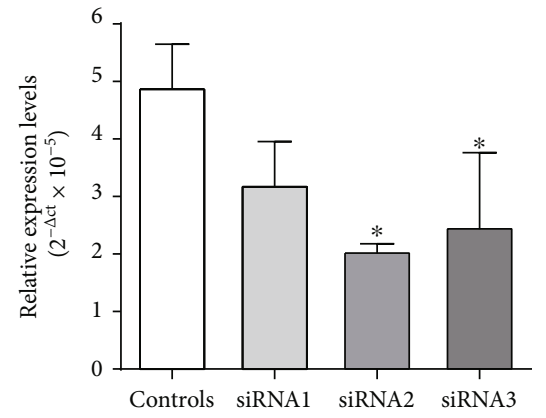

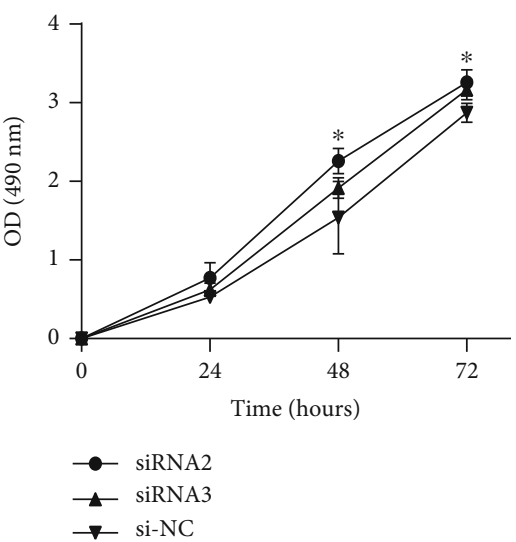

(c)
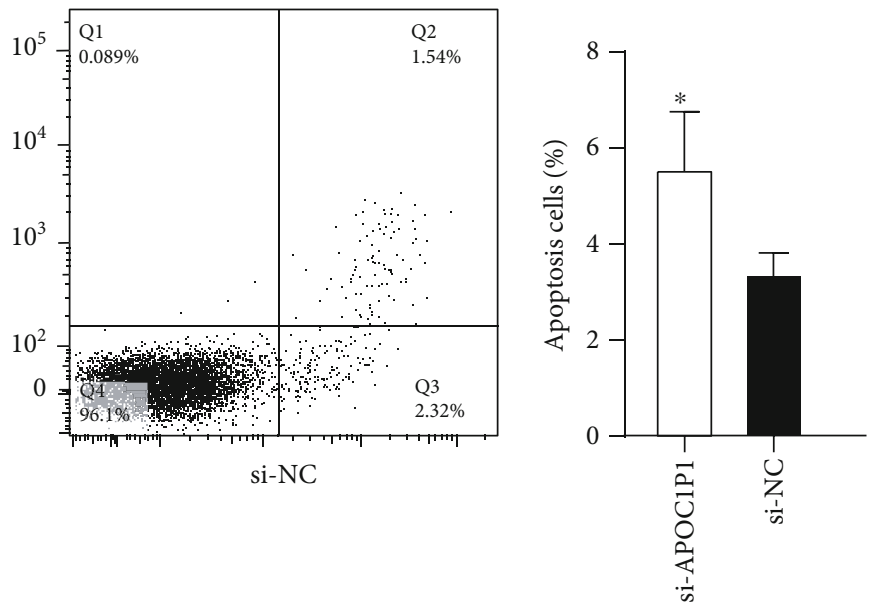

(d)
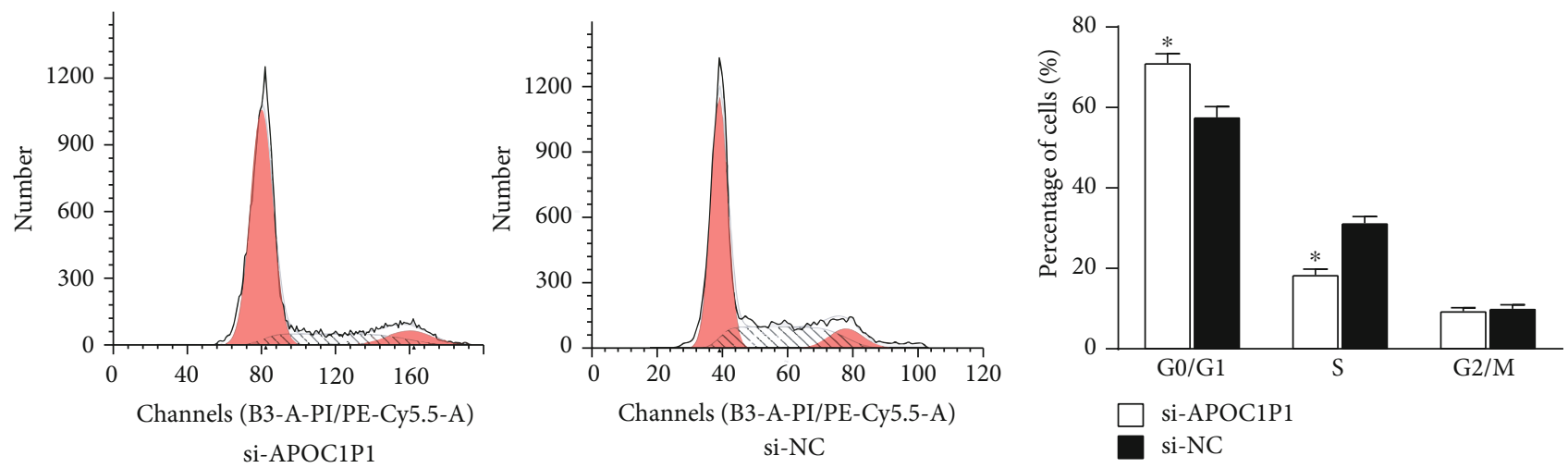

$\square \mathrm{G} 1$

$\square \mathrm{G} 2$

$\nabla \mathrm{S}$

(e)

FIgUre 3: Continued. 


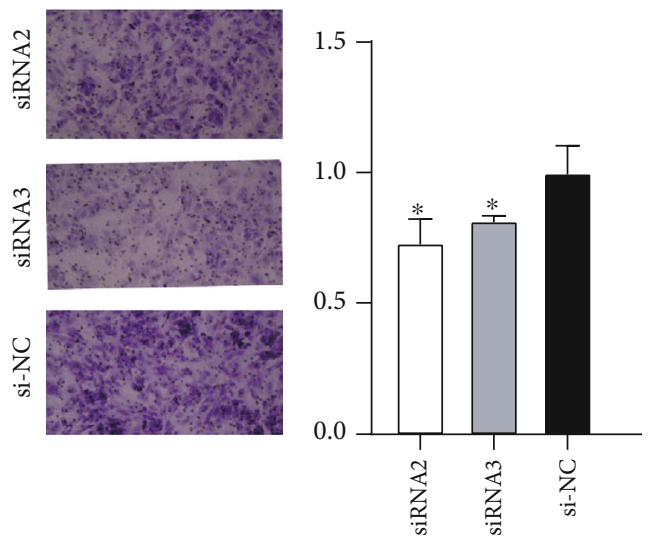

(f)
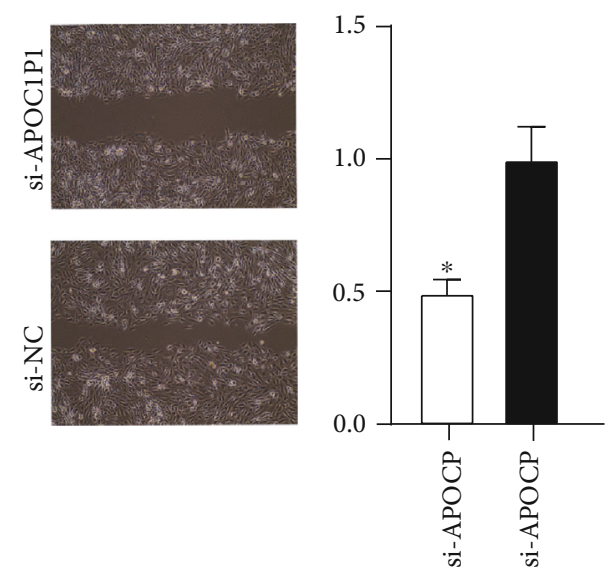

(g)

FIGURE 3: Effect of LncRNA APOC1P1 knockdown on proliferation, apoptosis, cell cycle, cell invasion, and migration capacity in ccRCC cells. (a) Expression of LncRNA APOC1P1 in human ccRCC cell lines (A498, 786-O, ACHN, and Caki-1) and an immortalized normal human renal tubular epithelial cell line (HK-2). (b) Validation of cell transfection efficiency of three siRNAs in A498 cells. (c) Cell proliferation measured by CCK-8 assays. (d, e) Cell apoptosis rate (d) and cell cycle (e) measured by flow cytometry. (f, g) Effect of LncRNA APOC1P1 knockdown on cell invasion and migration measured by Transwell chamber assays (f) and wound healing assays $(\mathrm{g})\left({ }^{*} P<0.05\right)$.

APOC1P1 expression was significantly associated with OS, but may not be an independent prognostic factor. LncRNA APOC1P1 plays an important role in the onset and progression of ccRCC by affecting cell proliferation and invasion. As a protooncogene, LncRNA APOC1P1 can be considered a prognostic biomarker and therapeutic target in ccRCC patients.

\section{Data Availability}

The data used to support the findings of this study are included within the article or the supplementary information file(s).

\section{Conflicts of Interest}

The authors declare no competing financial interests.

\section{Authors' Contributions}

Chong Sun and Zhonghan Zhou contributed equally to this work.

\section{Acknowledgments}

This study was partly funded by the National Natural Science Foundation of China (Grant No. NSFC 81502195) and the Medicine and Health Science Technology Development Project of Shandong Province (No. 2016WS0258). The authors thank Alison Sherwin, PhD, from Liwen Bianji, Edanz Group China (http://www.liwenbianji.cn/ac), for editing the English text of a draft of this manuscript.

\section{References}

[1] F. Bray, J. Ferlay, I. Soerjomataram, R. L. Siegel, L. A. Torre, and A. Jemal, "Global cancer statistics 2018: GLOBOCAN esti- mates of incidence and mortality worldwide for 36 cancers in 185 countries," CA: A Cancer Journal for Clinicians, vol. 68, no. 6, pp. 394-424, 2018.

[2] A. Znaor, J. Lortet-Tieulent, M. Laversanne, A. Jemal, and F. Bray, "International variations and trends in renal cell carcinoma incidence and mortality," European Urology, vol. 67, no. 3, pp. 519-530, 2015.

[3] B. Ljungberg, K. Bensalah, S. Canfield et al., "EAU guidelines on renal cell carcinoma: 2014 update," European Urology, vol. 67, no. 5, pp. 913-924, 2015.

[4] M. Wang, T. Huang, G. Luo et al., "Long non-coding RNA MEG3 induces renal cell carcinoma cells apoptosis by activating the mitochondrial pathway," Journal of Huazhong University of Science and Technology [Medical Sciences], vol. 35, no. 4, pp. 541-545, 2015.

[5] S. Xue, Q. W. Li, J. P. Che, Y. Guo, F. Q. Yang, and J. H. Zheng, "Decreased expression of long non-coding RNA NBAT-1 is associated with poor prognosis in patients with clear cell renal cell carcinoma," International Journal of Clinical and Experimental Pathology, vol. 8, no. 4, pp. 3765-3774, 2015.

[6] Y. Li, T. Wang, Y. Li et al., "Identification of long-non coding RNA UCA1 as an oncogene in renal cell carcinoma," Molecular Medicine Reports, vol. 13, no. 4, pp. 3326-3334, 2016.

[7] S. H. Rossi, T. Klatte, J. Usher-Smith, and G. D. Stewart, "Epidemiology and screening for renal cancer," World Journal of Urology, vol. 36, no. 9, pp. 1341-1353, 2018.

[8] J. Jia, F. Li, X. S. Tang et al., "Long noncoding RNA DANCR promotes invasion of prostate cancer through epigenetically silencing expression of TIMP2/3," Oncotarget, vol. 7, no. 25, pp. 37868-37881, 2016.

[9] X. Li, Z. Wu, X. Fu, and W. Han, "IncRNAs: insights into their function and mechanics in underlying disorders," Mutation Research/Reviews in Mutation Research, vol. 762, pp. 1-21, 2014.

[10] H. Su, H. Wang, G. Shi, H. Zhang, F. Sun, and D. Ye, "Downregulation of long non-coding RNA ENSG00000241684 is associated with poor prognosis in advanced clear cell renal cell 
carcinoma," European Journal of Surgical Oncology, vol. 44, no. 6 , pp. 840-846, 2018.

[11] S. Xu, X. M. Yi, C. P. Tang, J. P. Ge, Z. Y. Zhang, and W. Q. Zhou, "Long non-coding RNA ATB promotes growth and epithelial-mesenchymal transition and predicts poor prognosis in human prostate carcinoma," Oncology Reports, vol. 36, no. 1, pp. 10-22, 2016.

[12] J. Song, J. Peng, C. Zhu et al., "Identification and validation of two novel prognostic lncRNAs in kidney renal clear cell carcinoma," Cellular Physiology and Biochemistry, vol. 48, no. 6, pp. 2549-2562, 2018.

[13] X. H. Liao, J. G. Wang, L. Y. Li et al., "Long intergenic noncoding RNA APOC1P1-3 inhibits apoptosis by decreasing $\alpha$-tubulin acetylation in breast cancer," Cell Death \& Disease, vol. 7, no. 5, p. e2236, 2016.

[14] R. Tahbaz, M. Schmid, and A. S. Merseburger, "Prevention of kidney cancer incidence and recurrence: lifestyle, medication and nutrition," Current Opinion in Urology, vol. 28, no. 1, pp. 62-79, 2018.

[15] M. C. Wong, W. B. Goggins, B. H. Yip et al., "Incidence and mortality of kidney cancer: temporal patterns and global trends in 39 countries," Scientific Reports, vol. 7, no. 1, p. 15698, 2017.

[16] T. Gutschner and S. Diederichs, "The hallmarks of cancer: a long non-coding RNA point of view," RNA Biology, vol. 9, no. 6 , pp. 703-719, 2012.

[17] X. Cui, X. Jing, C. Long, J. Tian, and J. Zhu, "Long noncoding RNA MEG3, a potential novel biomarker to predict the clinical outcome of cancer patients: a meta-analysis," Oncotarget, vol. 8, no. 12, pp. 19049-19056, 2017.

[18] D. Kuang, X. Zhang, S. Hua, W. Dong, and Z. Li, "Long noncoding RNA TUG1 regulates ovarian cancer proliferation and metastasis via affecting epithelial-mesenchymal transition," Experimental and Molecular Pathology, vol. 101, no. 2, pp. 267-273, 2016.

[19] W.-p. Su, L.-n. Sun, S.-l. Yang et al., “Apolipoprotein C1 promotes prostate cancer cell proliferation in vitro," Journal of Biochemical and Molecular Toxicology, vol. 32, no. 7, 2018.

[20] H. L. Ko, Y. S. Wang, W. L. Fong, M. S. Chi, K. H. Chi, and S. J. Kao, "Apolipoprotein C1 (APOC1) as a novel diagnostic and prognostic biomarker for lung cancer: a marker phase I trial," Thorac Cancer, vol. 5, no. 6, pp. 500-508, 2014.

[21] Y. Sun, J. Zhang, F. Guo et al., "Identification of apolipoprotein C-I peptides as a potential biomarker and its biological roles in breast cancer," Medical Science Monitor, vol. 22, pp. 11521160, 2016.

[22] S. Takano, H. Yoshitomi, A. Togawa et al., "Apolipoprotein C1 maintains cell survival by preventing from apoptosis in pancreatic cancer cells," Oncogene, vol. 27, no. 20, pp. 2810-2822, 2008.

[23] R. Vikram, R. Ramachandran, and K. S. M. Abdul, "Functional significance of long non-coding RNAs in breast cancer," Breast Cancer, vol. 21, no. 5, pp. 515-521, 2014. 


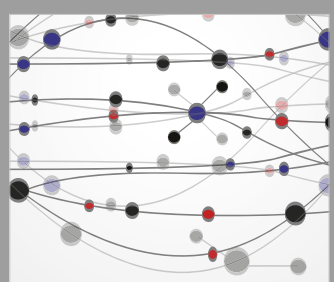

The Scientific World Journal
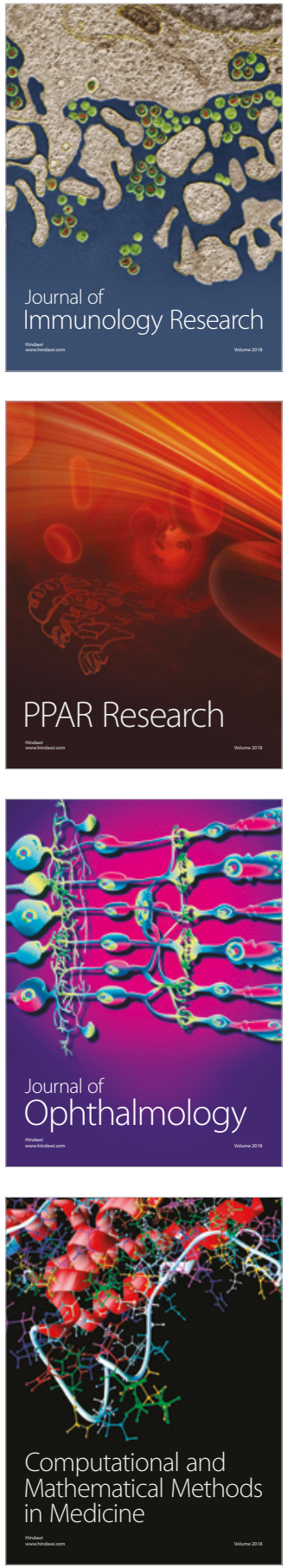

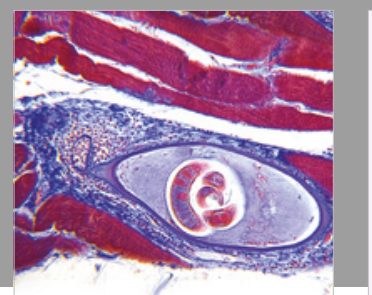

Gastroenterology Research and Practice

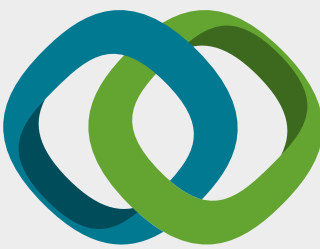

\section{Hindawi}

Submit your manuscripts at

www.hindawi.com
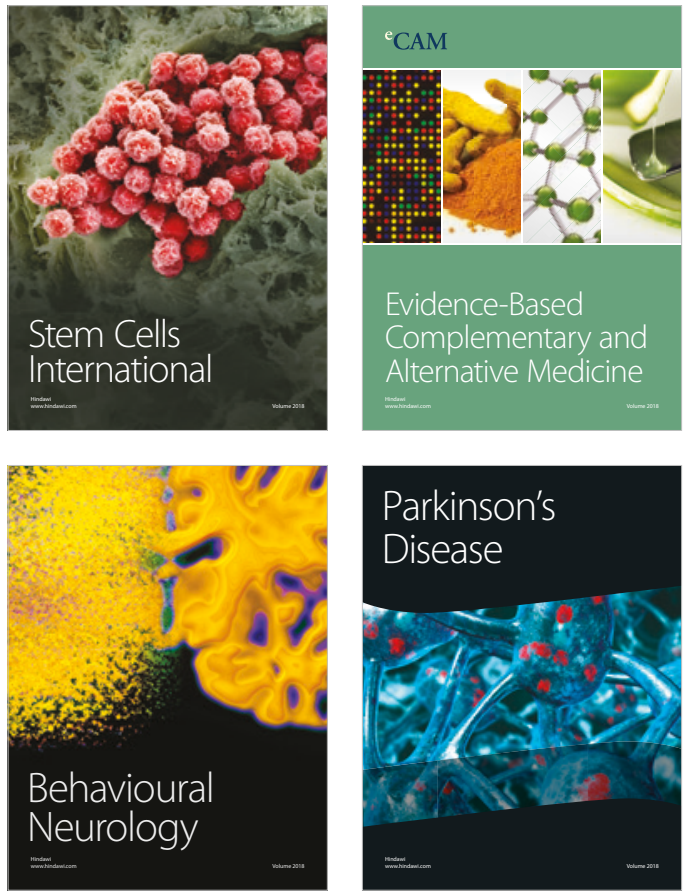

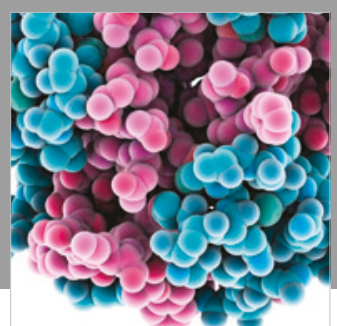

ournal of

Diabetes Research

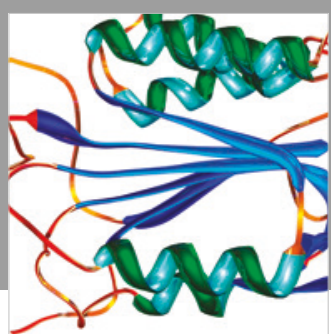

Disease Markers
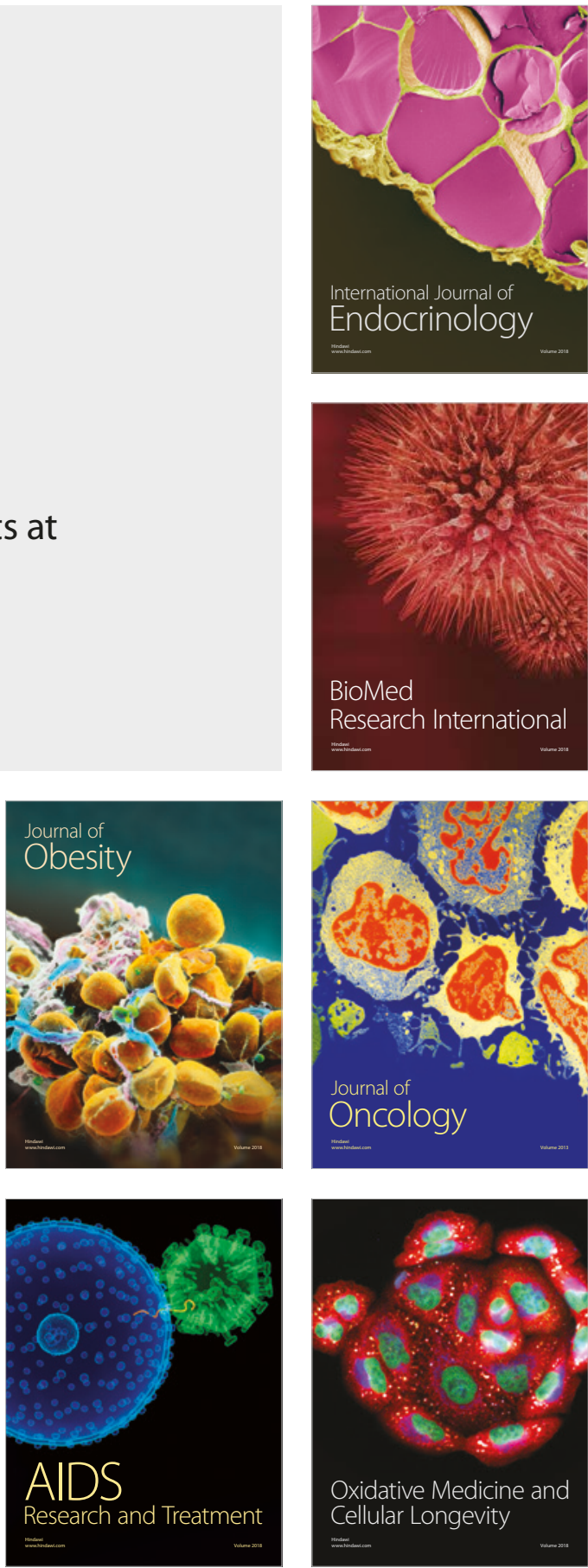